\title{
Ubiquitin Carboxyl-Terminal Hydrolase 7
}

National Cancer Institute

\section{Source}

National Cancer Institute. Ubiquitin Carboxyl-Terminal Hydrolase 7. NCI Thesaurus. Code C118239.

Ubiquitin carboxyl-terminal hydrolase 7 (1102 aa, 128 kDa) is encoded by the human USP7 gene. This protein is involved in catalyzing protein deubiquitination. 